\title{
Taurine and vascular tone modulation
}

\author{
Adrian Roşca ${ }^{1,2^{*}}$, Suzana Elena Voiculescu ${ }^{1 *}$, Bogdan Ovidiu Popescu ${ }^{3}$, Ana-Maria Zăgrean ${ }^{1}$
}

1"Carol Davila" University of Medicine and Pharmacy, Department of Functional Sciences, Discipline of Physiology and Fundamental Neuroscience, Bucharest, Romania

2"Victor Babeș” National Institute of Research-Development in the Pathology Domain, Bucharest, Romania

3"Carol Davila" University of Medicine and Pharmacy, Department of Neurology,

"Colentina" Clinical Hospital, Bucharest, Romania

*These authors contributed equally to this study

\section{ABSTRACT}

Taurine, an ubiquitary amino acid in the organism, has important physiological functions like bile salt formation, neuronal excitability regulation, immunomodulation, electrolytes homeostasis preservation, osmoregulation, membrane stabilisation, apoptosis inhibition and cell protection. This review article is focusing on the complex vasoactive influence of the sulphur-amino acid and on the possibly involved mechanisms that may underline these effects. Acting on endothelial and smooth muscle cells in the vessels, it regulates blood pressure and delays atherogenesis process, having an increased therapeutic potential that needs to be further explored.

Key words: taurine, vascular tone, vascular reactivity, nitric oxide, vascular smooth muscle cells

\section{INTRODUCTION}

Taurine (2-aminoethanesulfonic acid) is the most abundant intracellular free amino acid in the organism, found both in cytosol and plasma, mostly at the level of the myocardium, brain, skeletal muscles, retina, neutrophils and platelets $(1,2)$. Taurine is considered a semi-essential amino acid, because even if is synthesized in the liver from cysteine or methionine in a vitamin B6dependent manner, its major source is the diet, being predominantly found in meat, eggs and sea food (3).Taurine has been intensively studied in the past years, for its clinical applications as supplemented pharmaconutrient, or as a conditionally-essential amino acid in some subjects in need for long-term parenteral nutrition (3).

Taurine is involved in many physiological processes like bile acids conjugation, regulation of neuronal excitability, immunomodulation, cellular cations homeostasis preservation, osmoregulation, membrane stabilisation, apoptosis inhibition and cell protection $(1,4)$. Taurine has beneficial systemic or
Corresponding author: Roșca Adrian, MD, PhD, Lecturer "Carol Davila" University of Medicine and Pharmacy, Department of Functional Sciences, Discipline of Physiology and Fundamental Neuroscience, 8 Eroii Sanitari Blvd., Bucharest 050474, Romania E-mail: adrian.r@umf.ro 
organ-focused effects on the brain, liver, kidney, eye and cardiovascular system (1).

This review focuses on the complex vascular effects of taurine. At this level, it acts in different key points, lowering different risk factors for cardiovascular disease. Thus, it has been shown that taurine can prevent atherosclerosis development by ameliorating lipid profile (5), lowering homocysteine plasmatic concentration (6), reducing intima inflammation and oxidative stress $(7,8)$, decreasing endothelial dysfunction, modulating vascular reactivity and inhibiting vascular muscle cells proliferation (9). More than that, taurine has a protective role in diabetes mellitus (10), reduces blood pressure (11) and modulates haemostasis, especially platelet function (12).

Overall, through its complex positive actions and the lack of adverse effects, taurine is a good candidate for therapeutic use as dietary supplement in cardiovascular disease prevention (8).

\section{Taurine effects on vascular reactivity}

Taurine lowers blood pressure by reducing vascular resistance $(13,14)$ and plasma levels of endothelin, neuropeptide $Y$ and thromboxane $B_{2}(15,16)$. It also regulates kinin-kalikrein system and brings down plasmatic concentration of norepinephrine and epinephrine after long-term administration in rats (17) and humans (18-20). Other mechanisms by which taurine may influence blood pressure and delay the onset and progression of the atherosclerosis process may be linked to its action on endothelial cells or on the vascular smooth muscle cells (VSMCs), as it will be further presented.

\section{Taurine effects on induced-vasoconstriction}

There are several lines of evidence regarding protective effects of taurine in exogenous inducedvasoconstriction. Therefore, taurine administration in endothelial cell cultures from the human umbilical vein (HUVEC) incubated in a conditionally monocytes environment from smokers, brought to normal nitric oxide (NO) and endothelin-1 levels (16). In vivo taurine administration reduced the inhibition of the endothelial-dependent vasodilator response induced by a single low-density lipoprotein (LDL) injection. In vitro studies on HUVEC exposed to oxidized-LDL and incubated with taurine showed a reduced inhibition of NO formation and a reduced increase of the level of alpha tumor-necrosis factor ( $\alpha$-TNF), malondialdehyde (MDA), lactate dehydrogenase (LDH) and ADMA (the endogenous competitive inhibitor of NO synthase). Through all these effects taurine proved to be efficient in providing protection against induced endothelial dysfunction (21).

However, most of the information regarding taurine's action on vascular reactivity comes from animal studies. Chronic oral supplementation of the sulphur-amino acid in drinking water showed an ex-vivo diminished contractile response to norepinephrine and $\mathrm{KCl}$ of endothelium-free or endothelium-intact aortic rings isolated from WKY rats (22). On the other hand, taurine had no effect on the contractile response determined by norepinephrine in vitro, although it reduced $\mathrm{KCl}$-induced contraction in arteries isolated from rabbit ears (23). Long-term taurine administration attenuated epinephrine induced vasoconstriction of the mesenteric artery rings in spontaneously hypertensive stroke prone (SHRSP) rats (24). Taurine showed an in vitro vasodilating response on porcine coronary arteries vasoconstricted by serotonin, $\mathrm{KCl}, \mathrm{CaCl} 2, \mathrm{U} 46619$ (a TBX A2 analogue), in a dose dependent manner (25). It also demonstrated an in vitro inhibitory effect on norepinephrine / $\mathrm{KCl}$ - induced vasoconstriction, when rat aortic rings have been incubated with both high (26) and low taurine doses (27).

Treating rats with beta-alanine (a frequently used substance for endogenous taurine deprivation) produced an enhancement of norepinephrine and $\mathrm{KCl}$ induced vasoconstriction on the endothelium-free aortic rings (28) and a higher contractile response to angiotensin II (29).

\section{Taurine effects on induced-vasodilatation}

Except for inhibiting vasoconstriction in response to different exogenous substances, taurine also exerts a positive influence on substance-induced vasodilatation. Short-term (14 days) and low doses ( $1.5 \mathrm{~g} /$ day) administration of the sulphur-amino acid in diabetes type I patients normalized arterial rigidity and showed a positive effect on flux-mediated vasodilatation (30). The same dose of taurine, but administered in a shorter period ( 5 days) on young smokers led to an ultrasonographic improvement of the endothelialdependent vasodilatation (16).

Chronic taurine administration in WKY rats led to a better acetylcholine-induced vasodilatation in endothelium-intact aortic rings (22). A similar endothelialdependent effect was registered in diabetic and hypercholesterolemic rats, in which taurine augmented the acetylcholine-induced aortic rings relaxation (31). Also, incubating isolated vascular tissue, like aortic rings from streptozocin-treated diabetic rats with taurine amplified the acetylcholine-stimulated vascular relaxation (32). 
Endogenous taurine deficiency determined by betaalanine administration, led to reduced vasodilator response of the rat endothelium-intact aortic rings, when exposed to supramaximal acetylcholine levels. Beta-alanine treatment also produced a lower sensitivity of the endothelial - denuded aortic rings to sodium nitroprusside (28). Taurine depletion led to a similar effect on the endothelium-intact or endothelium-free aortic rings, reducing the 2-chloride-adenosine and 5'$\mathrm{N}$-ethil-carboxyamino-adenosine (adenosine receptors agonists) induced relaxation. Same action of the sulphur-amino acid was obtained when 2-chloride adenosine (but not 5'-N-ethil-carboxyamino-adenozine) has been used to induce vasodilatation in the superior mesenteric artery rings, suggesting that its vasoactive influence is independent on the artery type, but dependent on type of adenosine receptors agonist involved (33). On the other hand, in vitro endogenous taurine deprivation by beta-alanine administration didn't change the influence of some vasoactive agents on vascular reactivity (29).

\section{Taurine and endothelial NO release}

Available data regarding the influence of taurine on NO endothelial release are conflictual, despite a considerable number of findings suggesting a stimulatory effect of the sulphur-amino acid on NO production.

Upregulation of NO endothelial release, with consecutive vasodilating effects, has been observed both in human and animal studies. Therefore, either in vivo taurine supplementation in humans after a single injection of native LDL-cholesterol, or in vitro taurine incubation of HUVEC exposed to oxidized LDL, led to a lower raise of ADMA and a lower NO decrease induced by endothelial dysfunction (21). A similar protective role of taurine in endothelial dysfunction has been shown after oral administration of the sulphur-amino acid in young smokers, determining a lower inhibitory effect on vasodilatation induced by vasoactive agents, while in vitro taurine incubation with HUVEC exposed to a conditional monocyte environment from smokers, normalized NO levels (16). Taurine also showed a stimulatory effect on nitrite/nitrate (NOx) production, as stable end-products of NO metabolism, when administered in HUVEC cultures (34). The same results regarding taurine's effect on NO production have been noticed in lab animals. Supplementation of the sulphuramino acid in drinking water determined an improvement of NO bioavailability in post-weaning protein restriction rats, and partially prevented blood pressure increase (35). Additionally, endogenous taurine deficit induced by beta-alanine in L-NAME rats, was shown to produce a lower NO synthesis (33).

However, other studies noted a lack of any effect of taurine on the nitric oxide production. In vitro administration of the NO - synthase inhibitor L-NAME did not modify aortic rings relaxation when incubated with taurine, indicating that the vasodilating effect of the sulphur-amino acid is not NO-dependent (26). An exvivo study on taurine treated rats ( $1 \%$ in drinking water) showed a neutral effect on both blood pressure and NO, respectively NO-synthase (NOS) plasma levels (15). Moreover, oral administration of taurine prevented blood pressure raise and plasma NO decrease in cyclosporine treated rats, but without reaching the statistical significance (36). Taurine given in the same concentration ( $1 \%$ in drinking water) led to a significant increase in NO and NOS plasma levels, but this effect wasn't confirmed by taurine deprivation with betaalanine (37). Even if intraperitoneal supplementation of taurine limited NO metabolites production in Wistar rats fed with a high fructose diet and induced insulin resistance syndrome, it had no significant influence on their plasma concentration when was administered in healthy animals (38).

Taurine also showed a downregulatory action on nitric oxide production, by reducing the final NO products level in certain situations associating their raise. Therefore, it lowered mRNA expression of endothelial NOS on aortic rings of diabetic rats, this suggesting a potential beneficial effect of taurine on endothelial dysfunction induced by diabetes mellitus (39). It has also been shown that taurine may decrease mRNA expression of inducible NOS (iNOS), neuronal NOS (nNOS) and nitrosative stress in human Schwann cells exposed to a hyperglycaemic environment, suggesting a potential treatment in diabetic neuropathy (40). Taurine administered $2 \%$ in drinking water had a restorative effect in a rat model of hepatic fibrosis (induced by simultaneous chronic iron load and ethanol), by decreasing the nitrosative stress, raising the thiols level and decreasing the hepatic accumulation of nitrates. Oxidized protein level was also lowered, this being demonstrated by downregulatory action on the antibodies against 4-hydroxinenol and 3-nitrotyrozine (41). More than that, taurine has proved efficiency in lowering NO production, oxygen reactive species, apoptosis and isolated hepatocytes necrosis in rats treated with lipopolysaccharides and antioxidant substances, pointing an anti-inflammatory role of the sulphur-amino acid in the systemic inflammatory syndrome (42). Anti-inflammatory and anti-fibrotic roles have also been noted in a pulmonary fibrosis model induced by bleomycin administration in mice. Taurine 
and niacin simultaneous oral treatment suppressed bleomycin-induced NO raise, by reducing iNOS gene expression in the lungs (43).

Controversies in the studies regarding the effect of taurine on NO release may have multiple causes: different hypertension models used, different individual pathological conditions in humans which may interfere with the endothelial function assessment, or the still limited number of models used to investigate endothelial dysfunction (L-NAME, ADMA). In order to get a clear view of the real modulator mechanisms, by which taurine exerts its action on endothelial level, concomitant cell cultures and endothelial arterial slices from different vascular sources should be used, and sample collection from intact animals should always considered. Doses and time administration (acute/chronic) heterogeneity, as well as the different pathways used for drug administration could also explain the large variability of the results.

\section{Taurine influence on vascular smooth muscle cells}

Regarding the modulator influence of taurine on vascular tone, it has been shown that the sulphuramino acid could act also on other mechanisms than the ones previously mentioned, like interference of the vascular smooth muscle cells (VSMCs) proliferation. Unfortunately, data on this topic is rather poor (4). In vitro studies based on the assessment of cell number in rat aorta VSMCs culture or $(3 \mathrm{H})$ thymidine incorporation in DNA, showed direct antiproliferative effects of the sulphur-amino acid. Indirect effects of taurine on VSMCs proliferation have also been evidenced, by evaluating the PDGF (platelet-derived growth factor) receptor dephosphorylation $(44,45)$. These results are also sustained by ex vivo studies, in which taurine was shown to lower neointimal hyperplasia and proliferation of VSMCs in rats suffering an iatrogenic carotid injury (46). Taurine demonstrated indirect effects on VSCMs proliferation by lowering angiotensin II vascular action and consecutive remodelling $(15,47)$. Moreover, taurine lowered high plasma homocysteine levels induced by exogenous substances and antagonized the pro-oxidative effects of homocysteine on VSMCs $(6,48)$.

The sulphur-amino acid may also influence the atherogenic process by other mechanisms, like prevention of endothelial adhesion and trans-endothelial migration of leucocytes, inhibition of adhesion molecules expression (ICAM-1) or downregulating the endothelial apoptosis $(49,50)$. Taurine proved a beneficial effect on HUVEC in exogenous induced necrosis mediated by neutrophils (51), and also demonstrated a positive effect on atherosclerosis progression by improving the lipid profile. Therefore, it was shown to lower the LDL-cholesterol oxidation, to stimulate the bile acids production, to reduce cholesterol hepatic reserve and to prevent the raise of plasma triglycerides, total cholesterol, LDL and VLDL, while reducing the HDL levels $(2,9)$. Also, studies focused on vascular disease have noted that taurine can prevent LDL cholesterol oxidation in rodents, by reducing lectin-like receptor for oxidized LDL, along with an attenuation on the endothelial dysfunction and inhibition of the atherosclerotic process $(52,53)$.

\section{CONCLUSION}

Despite the conflictual literature data regarding its effect on endothelial function modulation, taurine has been proven to have an overall protective action on blood vessels (34). By integrating available data, we can conclude that taurine has an inhibitory influence on the vessel tone under basal conditions or under vasoactive stimulation, facilitating vasodilatation induced by exogenous substances, and inhibiting the induced vasoconstriction.

Taurine also contributes to a reduction of the smooth muscle cells proliferation in the arterial wall and has a positive effect on lipid profile, slowing down the atherosclerotic process. There are a series of intrinsic elementary mechanisms through which taurine has these complex protective effects. Even though there is a lot of available data in the literature concerning this subject, more studies are necessary to complete knowledge in this field.

\section{Acknowledgements}

This paper is partly supported by the Sectorial Operational Programme Human Resources Development (SOPHRD), financed by the European Social Fund and the Romanian Government under the contract number POSDRU 141531.

\section{REFERENCES}

1. Schaffer SW, Jong CJ, Ramila KC, Azuma J. Physiological roles of taurine in heart and muscle. J Biomed Sci. 2010 Aug 24;17 Suppl 1:S2.

2. Xu YJ, Arneja AS, Tappia PS, Dhalla NS.The potential health benefits of taurine in cardiovascular disease. Exp Clin Cardiol. 2008;13(2):57-65.

3. Lourenço R, Camilo ME. Taurine: a conditionally essential amino acid in humans? An overview in health and disease. Nutr Hosp. 2002 Nov-Dec; 17(6):262-70.

4. Abebe W, Mozaffari MS. Role of taurine in the vasculature: an overview of experimental and human studies. Am J Cardiovasc Dis. 2011;1(3):293311. Epub 2011 Sep 10.

5. Yamori Y, Murakami S, Ikeda K, Nara Y. Fish and lifestyle-related disease prevention: experimental and epidemiological evidence for anti-atherogenic 
potential of taurine. Clin Exp Pharmacol Physiol. 2004;31Suppl 2:S20-3.

6. Ahn CS. Effect of taurine supplementation on plasma homocysteine levels of the middle-aged Korean women. Adv Exp Med Biol. 2009;643:415-22.

7. Marcinkiewicz J, Kontny E. Taurine and inflammatory diseases. Amino Acids. 2014 Jan; 46(1):7-20.

8. Zulli A. Taurine in cardiovascular disease. Curr Opin Clin Nutr Metab Care. 2011 Jan;14(1):57-60.

9. Murakami S. Taurine and atherosclerosis. Amino Acids. 2014 Jan;46(1): 73-80.

10. Ito T, Schaffer SW, Azuma J. The potential usefulness of taurine on diabetes mellitus and its complications. Amino Acids. 2012 May;42(5):1529-39.

11. Militante JD, Lombardini JB. Treatment of hypertension with oral taurine: experimental and clinical studies. Amino Acids. 2002;23(4):381-93.

12. Hayes KC, Pronczuk A, Addesa AE, Stephan ZF. Taurine modulates platelet aggregation in cats and humans. Am J Clin Nutr. 1989 Jun;49(6):1211-6.

13. Meldrum MJ, Tu R, Patterson T, Dawson R Jr, Petty T. The effect of taurine on blood pressure, and urinary sodium, potassium and calcium excretion. Adv Exp Med Biol. 1994;359:207-15.

14. Anuradha CV, Balakrishnan SD.Taurine attenuates hypertension and improves insulin sensitivity in the fructose-fed rat, an animal model of insulin resistance. Can J Physiol Pharmacol. 1999 0ct;77(10):749-54.

15. Hu J, Xu X, Yang J, Wu G, Sun C, Lv Q. Anthypertensive effect of taurine in rat. Adv Exp Med Biol. 2009;643:75-84.

16. Fennessy FM, Moneley DS, Wang JH, Kelly CJ, Bouchier-Hayes DJ. Taurine and vitamin $C$ modify monocyte and endothelial dysfunction in young smokers. Circulation. 2003 Jan 28;107(3):410-5

17. Yamamoto J, Akabene S, Yoshimi H, Makai M, Ikeda M. Effects of taurine on stress-evoked hemodynamic and plasma catecholamine changes in spontaneously hypertensive rats. Hypertension. 1985 Nov-Dec;7(6 Pt 1):913-22.

18. Fujita $T$, Ando $K$, Noda $H$, Ito $Y$, Sato $Y$. Effects of increased adrenomedullary activity and taurine in young patients with borderline hypertension. Circulation. 1987 Mar;75(3):525-32.

19. Tanabe Y, Urata H, Kiyonaga A, Ikeda M, Tanaka H, Shindo M, Arakawa K. Changes in serum concentrations of taurine and other amino acids in clinical antihypertensive exercise therapy. Clin Exp Hypertens A. 1989;11(1): 149-65.

20. Nandhini AT, Anuradha CV. Hoe 140 abolishes the blood pressure lowering effect of taurine in high fructose-fed rats. Amino Acids. 2004 Jun;26(3): 299-303. Epub 2003 May 28.

21. Tan B, Jiang DJ, Huang H, Jia SJ, Jiang JL, Hu CP, Li YJ. Taurine protects against low-density lipoprotein-induced endothelial dysfunction by the DDAH/ADMA pathway. Vascul Pharmacol. 2007 May;46(5):338-45. Epub 2007 Jan 19.

22. Abebe W, Mozaffari MS. Effects of chronic taurine treatment on reactivity of the rat aorta. Amino Acids. 2000;19(3-4):615-23.

23. Franconi F, Giotti A, Manzini S, Martini F, Stendardi I, Zilletti L. The effect of taurine on high potassium- and noradrenaline-induced contraction in rabbit ear artery. Br J Pharmacol. 1982 Apr;75(4):605-12.

24. Li N, Sawamura M, Nara Y, Ikeda K, Yamori Y. Direct inhibitory effects of taurine on norepinephrine-induced contraction in mesenteric artery of stroke-prone spontaneously hypertensive rats. Adv Exp Med Biol. 1996;403:257-62.

25. Liu Y, Niu L, Zhang W, Cui L, Zhang X, Liang Y, et al. Effects of taurine on contractions of the porcine coronary artery. Pharmacol Rep. 2009 JulAug;61(4):681-9.

26. Niu LG, Zhang MS, Liu Y, Xue WX, Liu DB, Zhang J, et al. Vasorelaxant effect of taurine is diminished by tetraethylammonium in rat isolated arteries. Eur J Pharmacol. 2008 Feb 2;580(1-2):169-74. Epub 2007 Oct 25.

27. Ristori MT, Verdetti J. Effects of taurine on rat aorta in vitro. Fundam Clin Pharmacol. 1991;5(3):245-58.

28. Abebe W, Mozaffari MS. Taurine depletion alters vascular reactivity in rats. Can J Physiol Pharmacol. 2003 Sep;81(9):903-9.

29. Mozaffari MS, Abebe W. Cardiovascular responses of the taurine depleted rat to vasoactive agents. Amino Acids. 2000;19(3-4):625-34.

30. Moloney MA, Casey RG, O'Donnell DH, Fitzgerald P, Thompson C Bouchier-Hayes DJ. Two weeks taurine supplementation reverses endothelial dysfunction in young male type 1 diabetics. Diab Vasc Dis Res. 2010 Oct;7(4):300-10.

31. Kamata K, Sugiura M, Kojima S, Kasuya Y. Restoration of endothelium dependent relaxation in both hypercholesterolemia and diabetes by chronic taurine. Eur J Pharmacol. 1996 May 6;303(1-2):47-53.

32. Abebe W. Effects of taurine on the reactivity of aortas from diabetic rats. Life Sci. 2008 Jan 30;82(5-6):279-89.

33. Abebe, W, Mozaffari MS. Effect of taurine deficiency on adenosine receptor-mediated relaxation of the rat aorta. Vascul Pharmacol. 2003 Nov;40(4):219-28.

34. Pasaoglu OT, Turkozkan N, Ark M, Polat B, Agilli M, Yaman H. The effect of taurine on the relationship between NO, ADMA and homocysteine in endotoxin-mediated inflammation in HUVEC cultures. Inflammation. 2014 Oct;37(5):1439-43.

35. Maia AR, Batista TM, Victorio JA, Clerici SP, Delbin MA, Carneiro EM, et al. Taurine supplementation reduces blood pressure and prevents endothelial dysfunction and oxidative stress in post-weaning protein-restricted rats. PLoS One. 2014 Aug 29;9(8):e105851.

36. Hagar $\mathrm{HH}$, El Etter E, Arafa M. Taurine attenuates hypertension and renal dysfunction induced by cyclosporine A in rats. Clin Exp Pharmacol Physiol. 2006 Mar;33(3):189-96.

37. Yang J, Wu G, Feng Y, Lv Q, Lin S, Hu J. Effects of taurine on male reproduction in rats of different ages. J Biomed Sci. 2010; 17 Suppl 1:S9.

38. El Mesallamy HO, El-Demerdash E, Hammad LN, El Magdoub HM. Effect of taurine supplementation on hyperhomocysteinemia and markers of oxidative stress in high fructose diet induced insulin resistance. Diabetol Metab Syndr. 2010 Jun 30;2:46.

39. Ikubo N, Saito M, Tsounapi P, Dimitriadis F, Ohmasa F, Inoue $S$, et al. Protective effect of taurine on diabetic rat endothelial dysfunction. Biomed Res. 2011 Jun;32(3):187-93.

40. Askwith T, Zeng W, Eggo MC, Stevens MJ. Taurine reduces nitrosative stress and nitric oxide synthase expression in high glucose-exposed human Schwann cells. Exp Neurol. 2012 Jan;233(1):154-62.

41. Devi SL, Anuradha CV. Oxidative and nitrosative stress in experimental rat liver fibrosis: Protective effect of taurine. Environ Toxicol Pharmacol. 2010 Mar;29(2):104-10.

42. Redmond HP, Wang JH, Bouchier-Hayes D. Taurine attenuates nitric oxideand reactive oxygen intermediate-dependent hepatocyte injury. Arch Surg. 1996;131:1280-7.

43. Gurujeyalakshmi G, Wang Y, Giri SN. Suppression of bleomycin-induced nitric oxide production in mice by taurine and niacin. Nitric Oxide. 2000 Aug;4(4):399-411.

44. Zhang X, Tenner TE Jr, Lombardini JB. Inhibition of rat vascular smooth muscle cell proliferation by taurine and taurine analogues. Biochem Pharmacol. 1999 Jun 1;57(11):1331-9.

45. Yoshimura $\mathrm{H}$, Nariai $\mathrm{Y}$, Terashima M, Mitani $\mathrm{T}$, Tanigawa $\mathrm{Y}$. Taurine suppresses platelet-derived growth factor (PDGF) BB-induced PDGF-beta receptor phosphorylation by protein tyrosine phosphatase-mediated dephosphorylation in vascular smooth muscle cells. Biochim Biophys Acta. 2005 Sep 30;1745(3):350-60

46. Murakami S, Sakurai T, Toda Y, Morito A, Sakono M, Fukuda N. Prevention of neointima formation by taurine ingestion after carotid balloon injury. Vascul Pharmacol. 2010 Sep-0ct;53(3-4):177-84.

47. Schaffer SW, Lombardini JB, Azuma J. Interaction between the actions of taurine and angiotensin II. Amino Acids. 2000;18(4):305-18.

48. Chang L, Xu JX, Zhao J, Pang YZ, Tang CS, Qi YF. Taurine antagonized oxidative stress injury induced by homocysteine in rat vascular smooth muscle cells. Acta Pharmacol Sin. 2004 Mar;25(3):341-6.

49. Casey RG1, Gang C, Joyce M, Bouchier-Hayes DJ. Taurine attenuates acute hyperglycemia-induced endothelial cell apoptosis, leucocyteendothelial cell interactions and cardiac dysfunction. J Vasc Res. 2007; 44(1):31-9. Epub 2006 Dec 11.

50. Egan BM, Chen G, Kelly CJ, Bouchier-Hayes DJ. Taurine attenuates LPSinduced rolling and adhesion in rat microcirculation. J Surg Res. 2001 Feb;95(2):85-91.

51. Wang JH, Redmond HP, Watson RW, Condron C, Bouchier-Hayes D. The beneficial effect of taurine on the prevention of human endothelial cell death. Shock. 1996 Nov;6(5):331-8.

52. Wang LJ, Yu YH, Zhang LG, Wang Y, Niu N, Li Q, et al. Taurine rescues vascular endothelial dysfunction in streptozocin induced diabetic rats: correlated with downregulation of LOX-1 and ICAM-1 expression on aortas. Eur J Pharmacol. 2008 Nov 12;597(1-3):75-80.

53. Chiba $Y$, Ando K, Fujita T. The protective effects of taurine against renal damage by salt loading in Dahl salt-sensitive rats. J Hypertens. 2002 Nov;20(11):2269-74. 